\title{
Modeling of the beam excited fishbone mode in EAST
}

Baolong $\mathrm{Hao}^{1,2^{*}}$, Roscoe White ${ }^{3}$, Xiang $\mathrm{Gao}^{1,4^{*}}$, Guo Meng ${ }^{5}$, Guoqiang $\mathrm{Li}^{4}$, Bin $\mathrm{Wu}^{4}$, Jinfang Wang ${ }^{4}$, Liqing $\mathrm{Xu}^{4}$, Yi Yuan ${ }^{4}$, Shen $\mathrm{Wei}^{4}$, EAST team

1. Advanced Energy Research Center, Shenzhen University, Shenzhen 518060,

\section{China}

2. Key Laboratory of Optoelectronic Devices and Systems of Ministry of Education and Guangdong Province, College of Optoelectronic Engineering, Shenzhen University, Shenzhen 518060, China

3. Princeton Plasma Physics Laboratory, Princeton University, Princeton, NJ 08543, USA

4. Institute of Plasma Physics, Chinese Academy of Sciences, Hefei 230031, China

5. Max Planck Institut für Plasmaphysik, Garching 85748, Germany

E-mail: blhao@szu.edu.cn, xgao@ipp.ac.cn

Abstract: Many beam related instabilities have been observed on EAST. This paper reports on detailed calculations of beam driven fishbone and sawtooth instabilities using a general fishbone dispersion relation. The ideal fluid contribution and eigenfunction were calculated with the MHD code NOVA, the kinetic contribution was evaluated with sample fast ion distributions in the guiding center code ORBIT, and the general inertial term was calculated using experiment data. The sawtooth branch was stabilized both by co-beam injection and balanced injection, but the stable domain for sawtooth and fishbone mode is wider for balanced injection. The fishbone of precessional branch is excited by both trapped and passing particles. To wider stable domain, we propose a modulated balanced beam heating scenario for long pulse high beta discharges in EAST, with the single beam pulse turn on time shorter than the plasma energy confinement time and longer than beam ion slowing down time.

Keywords: dispersion relation, sawtooth, fishbone, stable regime, kinetic contribution

\section{Introduction}

One of outstanding prerequisites for magnetic confinement fusion energy is that the energetic particle population be well confinement before thermalization. Although fast ions can improve plasma confinement through sustaining a transport barrier in some circumstances, energetic particles can substantially influence the bulk plasma MHD behavior. Fast ions are produced by fusion reactions and through auxiliary heating, such as energetic neutral atom injection. As a primary heating method in the EAST tokamak, beam injection produced excellent plasma heating and current drive, and also many instabilities related to energetic particles. Neutral Beam Injection (NBI) is the standard method for energetic particle physics research until a burning plasma experiment is produced in a fusion reactor. The safety factor profile in one operation scenario for a fusion reactor has a minimum $q$ value below unity, giving the possibility for internal kink modes, having two branches, sawtooth and fishbone [1].

The sawtooth is a periodic relaxation and oscillation of plasma center parameters, and decades of research reveals that frequent small amplitude sawtooth can help 
remove impurities from the plasma center, and should benefit the helium ash problem in a fusion reactor. Longer period sawtooth oscillations have the potential to couple with and trigger NTM and ELMs, which degrade plasma confinement, and obviously, a monster sawtooth period can roughly defined as longer than the local energy confinement time [2]. The fishbone is a high frequency repetitive oscillation, driven unstable by the free energy from the gradient of the fast ion distribution or the bulk ions, modeled with the wave particle resonance and a finite diamagnetic frequency [3, 18]. Both the sawtooth oscillation and the fishbone instability have $n=1, m=1$ internal kink mode structure dominantly, and the numerical evolution of these instabilities has been carried out with a self-consistent hybrid model. To investigate the nonlinear dynamics during a fishbone cycle and other energetic particle modes self-consistently, two initial value codes have been used in analysis of EAST experiments, M3D-K and MEGA, and reproduce results such as frequency chirps, fast ion redistribution and beam parameter dependence $[4,5]$. This paper investigates the fishbone with the same experimental data through a variational energy principle method, including the singular layer's contribution in the internal kink dispersion relation. Generally, the instability's growth time is much shorter than the energy confinement time, it is more important to determine the mode stable-unstable regime than to calculate the exact mode growth rate [6].

Section 2 presents the fishbone shot data in EAST and its beam injection system, section 3 describes the general fishbone dispersion relation and its analytical solution, section 4 gives the numerical results with different beam particle distributions and finds a stable window for sawtooth and fishbone, section 5 studies the fast ion loss with the measured perturbation amplitude and frequency with a guiding center simulation, the last section summarizes the paper and proposes a beam heating scenario in EAST with balanced injection.

\section{Fishbone shot in EAST experiment}

The EAST tokamak is a good platform to investigate energetic particle modes since it has a flexible beam injection scenario and an upgraded diagnostic system. NBI has four positive deuterium beam sources, injected co and counter to the plasma current with full energy 40-80keV, 1-4 MW in each ion source. The tangency radii of the four independent beam injectors are $126.082 \mathrm{~cm}$ of co-tangent, $733.330 \mathrm{~cm}$ of co-perp, $-609.050 \mathrm{~cm}$ of counter-perp, $-113.818 \mathrm{~cm}$ of counter-tangent. The plan view of the initial beam distribution for the different injectors is shown in figure 1, and the deposition function was calculated using the TRANSP/NUBEAM code. All the beam lines are flat in the horizontal plane of the machine which means on-axis injection. With the flexible poloidal field control system, EAST also has the potential to carry out off-axis beam heating.

The details of the fishbone shot analyzed in this paper have been reported in reference [7]. The plasma current $\mathrm{Ip}=400 \mathrm{kA}$ is counter clockwise viewed from the top, the toroidal magnetic field $\mathrm{B}_{\mathrm{T}}=1.85 \mathrm{~T}$ is in the same direction, although also a clockwise direction can be obtained, other main plasma parameter is shown in table 1.

The electron temperature and density at plasma center are about $T_{e}(0)=2.5 \mathrm{keV}$, 
$n e(0)=5 \times 10^{19} \mathrm{~m}^{-3}$, the plasma profiles measured by XCS shown in fig 2 . During the fishbone burst from 4.6 to $4.72 \mathrm{~s}, 3 \mathrm{MW}$ beam power was injected into the tokamak inner vessel, with energy and pitch angle distributions of co injected beam ions shown in fig 3, obtained from a TRANSP/NUBEAM classical slowing down calculation [8].

The primary diagnostics for the fishbone instability are the soft x-ray imaging system, the neutron fluence detector and Mirnov coils. There is a typical top hat displacement pattern of the 1/1 internal kink mode, and frequency whistling down during the cycle. The characteristic fishbone frequency chirps down from $15-20 \mathrm{kHz}$, several cycles of fishbone onset and decay are shown in fig 4 . This paper investigates the general fishbone dispersion relation numerically and calculates induced fast ion loss with the guiding center code ORBIT which can give bounce orbit averaging over the fast ion distribution.

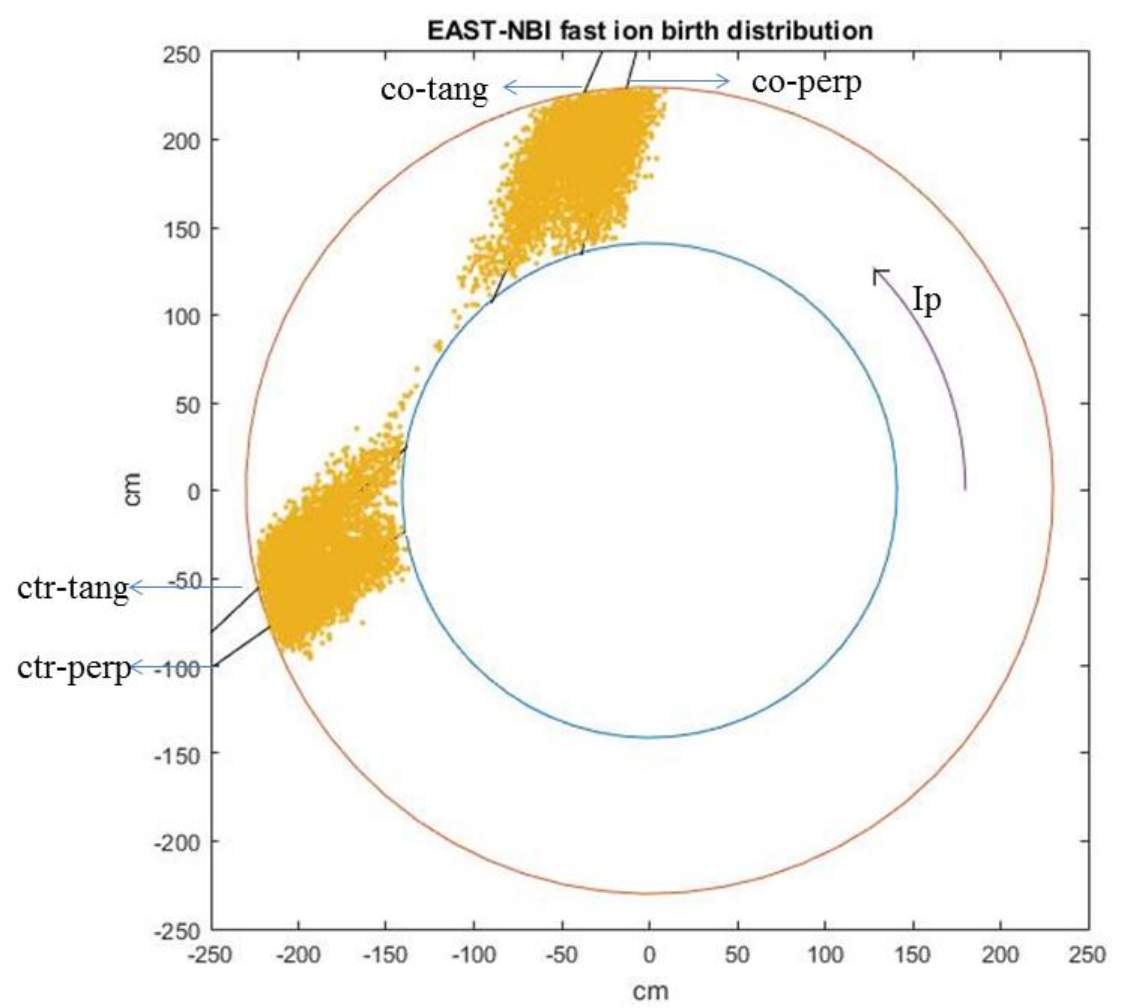

Figure 1. Fast ion initial deposition from four beam injectors in EAST plasma with bird's view.

Table 1. Plasma parameters of fishbone shot in EAST 48605.

\begin{tabular}{ll}
\hline Plasma current & $\mathrm{I}_{\mathrm{p}}=400 \mathrm{kA}$ (counter-clockwise) \\
Toroidal magnetic field on plasma center & $\mathrm{B}_{\mathrm{T}}=1.85 \mathrm{~T}$ (counter-clockwise) \\
Major and minor plasma radius & $\mathrm{R}_{0}=1.92 \mathrm{~m}, \mathrm{a}=0.45 \mathrm{~m}$ \\
Elongation and triangularity & $\kappa=1.5, \delta=0.488$ \\
Edge safety factor & $\mathrm{q} 95 \sim 5$ \\
Center safety factor & $\mathrm{q} 0 \sim 0.7-0.9$ \\
Plasma shape & $\mathrm{LSN}$ \\
Beam power injected & $\mathrm{P}_{\mathrm{inj}}=3 \mathrm{MW}$ \\
Beam energy & $\mathrm{E}_{\mathrm{nb}}=62 \mathrm{keV}$ \\
\hline
\end{tabular}



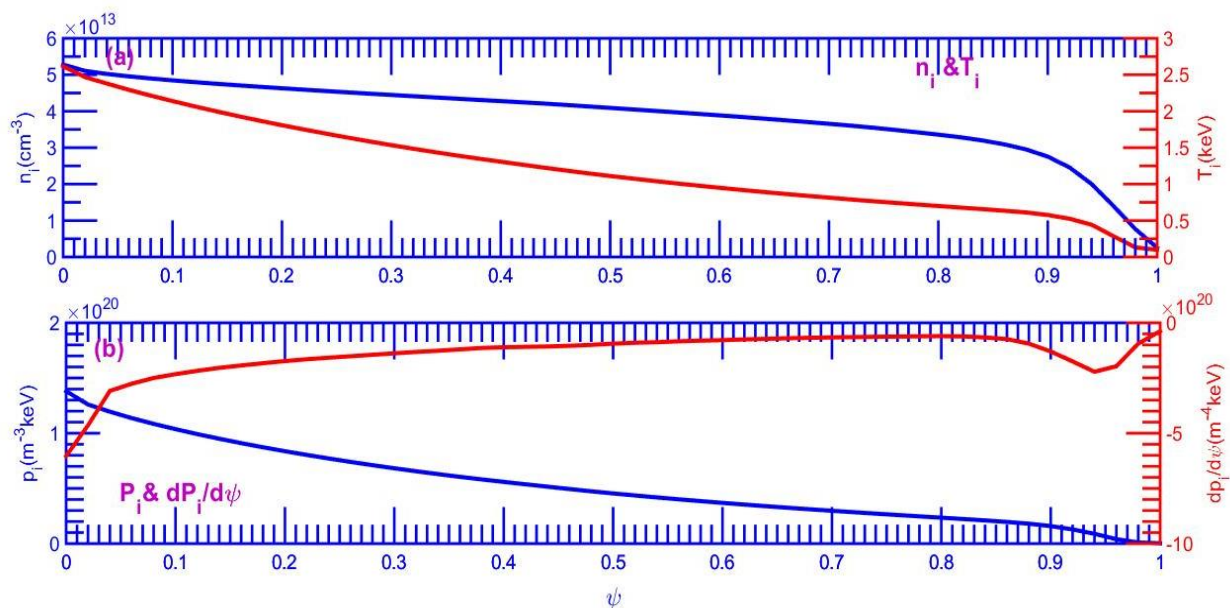

Figure 2. Plasma profiles of (a) density, temperature, and (b) pressure in EAST fishbone shot 48605 .

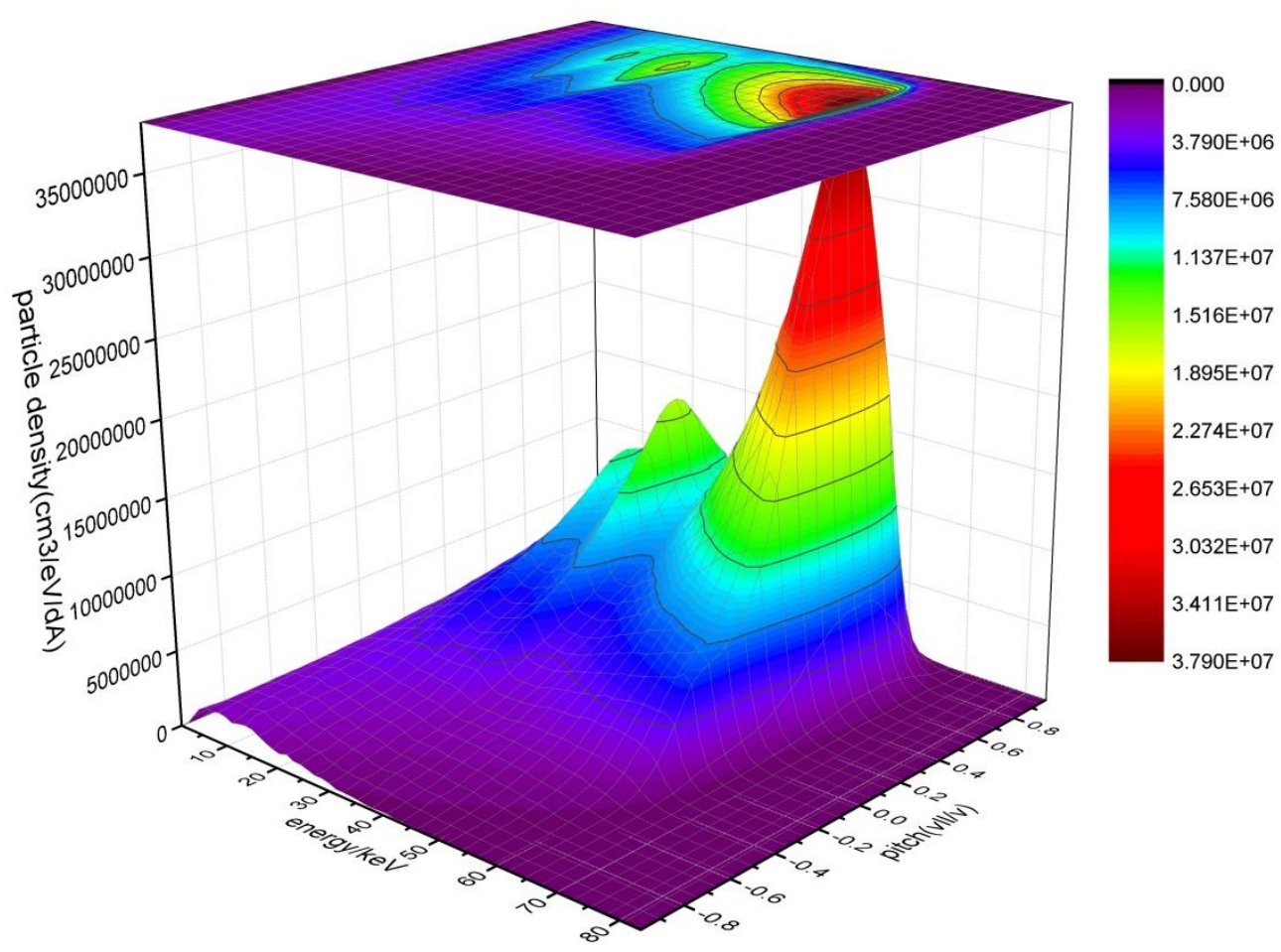

Figure 3. Energy and pitch angle distribution of co-injected beam ions in fishbone shot 48605 . 

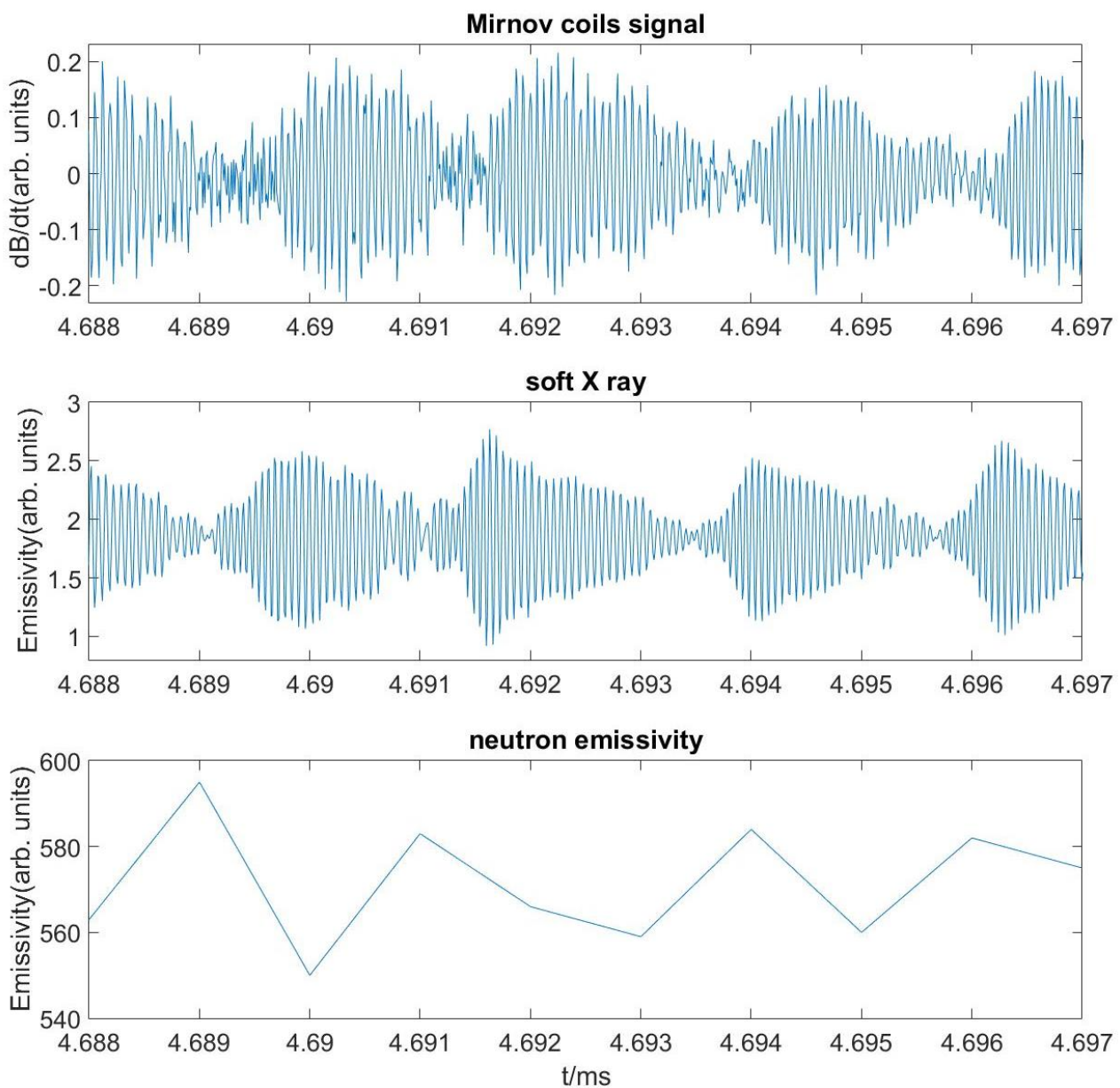

Figure 4. Time evolution of the Mirnov signals, soft x-ray and neutron emission in EAST fishbone shot 48605.

\section{Dispersion relation of internal kink instability and analytical solutions}

The general fishbone dispersion relation, mainly developed by Chen and Coppi is $D(\xi)=0=I+\delta W_{M H D}+\delta W_{k}$, have two types of modes, the energetic particle continuum mode and the discrete Alfven gap mode $[9,18,19]$. The dispersion relation includes three terms, the inertial contribution $I$ from bulk ions, a fluid contribution $\delta W_{M H D}$ from the thermal plasma, the fast ion kinetic contribution $\delta W_{k}$. As a typical energetic particle mode, fishbone have two branches, one is high frequency oscillation which determined by resonant fast ion's characteristic drift frequency, usually the toroidal precession frequency on tokamak. It is often called precessional fishbone and has a critical fast ion beta to destabilization. Another fishbone branch is called diamagnetic branch, which have a low frequency related to the diamagnetic frequency evaluated at the $\mathrm{q}=1$ surface, its free energy is the radial gradient of bulk ions and a 'viscous' dissipative process is necessary. In the case of averaged precession frequency and diamagnetic frequency are not well separated, it is difficult to distinguish them in the experiment.

When modeling the fishbone, the inertial term mainly comes from the $\mathrm{q}=1$ singular 
surface of the $n=1$ internal kink mode, its expression taking account of resistivity and diamagnetic frequency is $\quad I=-\frac{8 i \Gamma\left(\left(\Lambda^{3 / 2}+5\right) / 4\right) \sqrt{\omega\left(\omega-\omega_{*}\right)}}{\Lambda^{9 / 4} \Gamma\left(\left(\Lambda^{3 / 2}-1\right) / 4\right) \omega_{A}}, \quad$ where $\Lambda=-i\left(\omega\left(\omega-\omega_{*_{\mathrm{e}}}\right)\left(\omega-\omega_{*_{\mathrm{i}}}\right)^{1 / 3} / \gamma_{R}, \quad \gamma_{R}=S^{-1 / 3} \omega_{A}\right.$ is the resistive growth rate, $S=\tau_{R} / \tau_{A}$ is the magnetic Reynolds number with $\tau_{R}=\mu_{0} r_{s}^{2} / \eta$ the characteristic resistive diffusion time, $\omega_{A}=V_{A} /\left(\sqrt{3} \mathrm{Rr}_{\mathrm{s}} \mathrm{q}^{\prime}\right)$ is the effective shear Alfven frequency, $r_{s}$ is the minor radius of the $q=1$ surface, and all terms in inertial contribution are evaluated at this singular layer[10].

With the plasma profiles and q profile shown in the fig 2 and fig 5 , the thermal ion diamagnetic frequency is determined as $\omega_{*_{\mathrm{i}}}=\frac{-1}{m_{i} n r \omega_{c i}} \frac{d p_{i}}{d r}=3 \mathrm{kHz}$, which is much smaller than fishbone frequency observed. The thermal electron diamagnetic frequency is assumed equal to $\omega_{*_{\mathrm{e}}}=-\omega_{*_{\mathrm{i}}}=-3 \mathrm{kHz}$. The shear Alfven frequency is determined as $\omega_{A}=5.44 * 10^{6} \mathrm{rad} / \mathrm{s}$ with the Alfven velocity $V_{A}=\frac{B}{\left(\mu_{0} \mathrm{n}_{\mathrm{i}} \mathrm{m}_{\mathrm{i}}\right)^{1 / 2}}=3.927 * 10^{6} \mathrm{~m} / \mathrm{s}$, the resistive time $\tau_{R}$ is $3.079 \mathrm{~s}$, the ratio of resistive time to Alfven time is $S=2.7 * 10^{6}$. For large value magnetic Reynolds number cases $S \geq 10^{10}$, the inertial term using the ideal limit but keeping the $\omega_{*_{\mathrm{i}}}$ effect can be expressed as $I=-i \sqrt{\omega\left(\omega-\omega_{* \mathrm{i}}\right)} / \omega_{A}$.

The fluid MHD potential energy change is generally real, after normalization by $2 R /\left[\pi\left(B r_{\mathrm{s}} \xi_{r 0}\right)^{2}\right]$ is $\delta W_{M H D}=-\gamma_{I} / \omega_{A}$, with $\gamma_{I}$ is the ideal MHD growth rate. With equilibrium and kinetic profiles of this fishbone shot, we find the ideal MHD eigenmode with the NOVA code, and find the $n=1$ ideal mode growth rate to be about $\gamma_{I}=27 \mathrm{kHz}$, which is much larger than diamagnetic frequency. The eigenmode corresponding to this internal kink eigenvalue is shown in fig 5. 


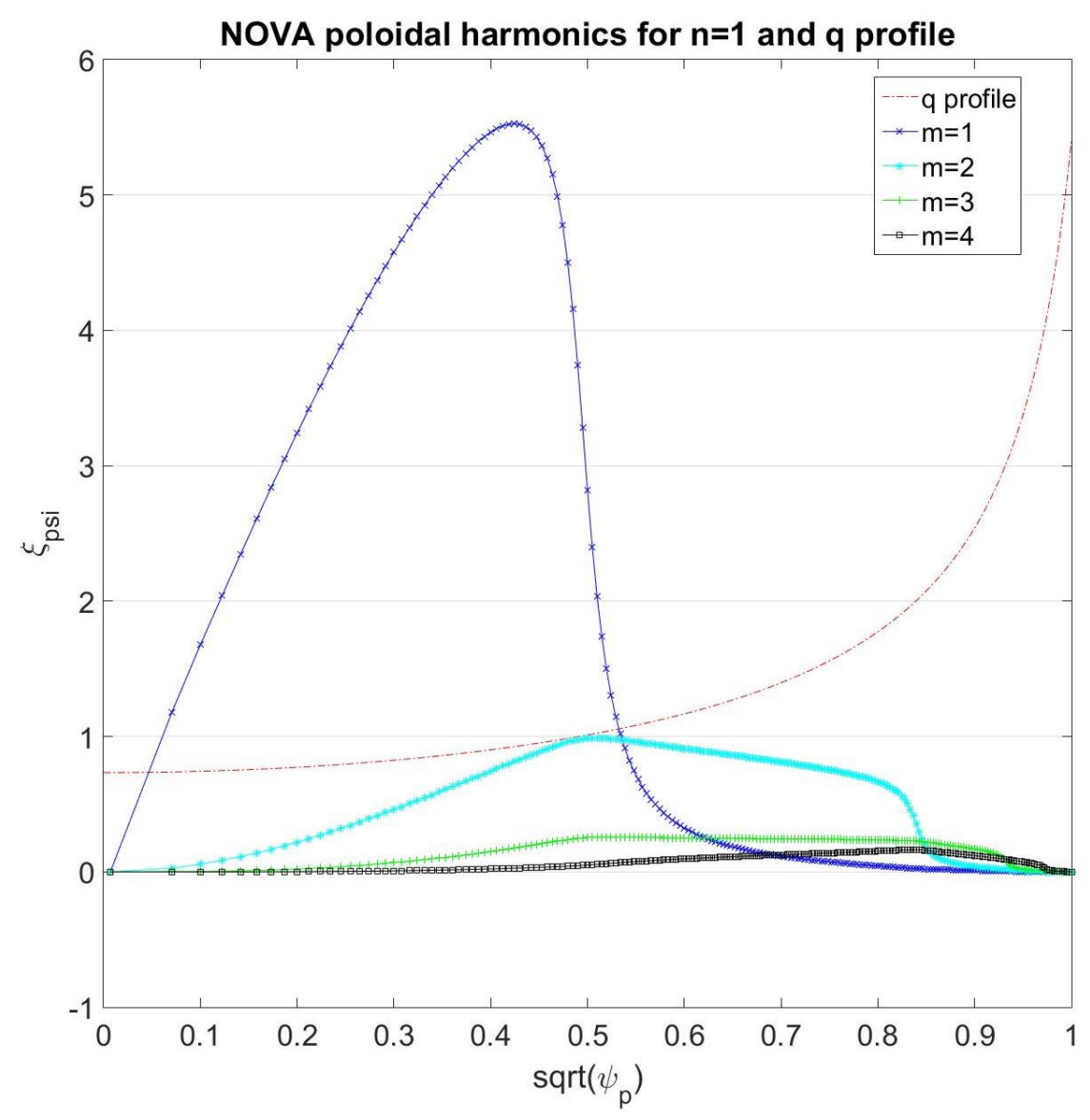

Figure 5. The poloidal components of $n=1$ internal kink mode eigenfunction and safety factor profile in fishbone shot 48605 , with total $\beta_{T}=0.038$.

Precessional fishbone is not an eigenmode of the bulk plasma but an EP-continuum mode, with real frequency mainly determined by the fast ion distribution. The fast ion potential energy change in this fishbone dispersion relation is $\delta \widehat{W}_{k}=\frac{-V \beta_{h}}{4 \pi^{2}\left(r_{\mathrm{s}} \xi\right)^{2} \sum_{j} E_{j}} \sum_{k}\left[\frac{\partial_{\Psi_{p}} F}{F} E H-\sum_{l} \frac{4 E^{2} Q}{F} \frac{\bar{J}^{*} \bar{J}}{\omega_{d}-\omega+(n q-l) \Omega_{\mathrm{t}}}\right]_{\mathrm{k}}$ in the Monte Carlo representation. The first term is the non-resonant contribution and is real, the second term is resonant contribution and is imaginary part of mode-particle interaction, where $H\left(\Psi_{\mathrm{p}}, \theta\right)=\left(\vec{\xi}_{\perp} \cdot \vec{\kappa}\right)\left(\vec{\xi}_{\perp} \cdot \nabla \Psi_{\mathrm{p}}\right), \quad \mathrm{J}\left(\Psi_{\mathrm{p}}, \theta\right)=(1-\mu B / E)\left(\vec{\xi}_{\perp} \cdot \vec{\kappa}\right), \quad Q=\omega \partial_{E} F-\partial_{\Psi_{p}} F$, and fast ion beta $\beta_{h}=8 \pi / B_{0}{ }^{2} V \int E d N$ [6]. The Monte Carlo representation of the fast ion distribution $F\left(\Psi_{p}, E, \mu\right)$ is convenient for the guiding center code ORBIT calculation, which can give the bounce averaged poloidal transit frequency $\Omega_{\mathrm{t}}=\overline{d \theta / d t}$ and toroidal precession frequency $\omega_{d}=\overline{d \zeta / d t}$. 
If the fast ion $\beta_{h}$ is relatively low, the kinetic contribution can be treated as a perturbation, then the non-resistive limit of the dispersion function $-i \sqrt{\omega\left(\omega-\omega_{* \mathrm{i}}\right)}-\gamma_{I}+\omega_{A} \delta \hat{W}_{k}=0$ is a quadratic equation for the complex frequency $\omega$, with roots depending on the discriminant $\gamma^{2}{ }_{I}-\omega^{2}{ }_{*_{\mathrm{i}}} / 4$. In this fishbone shot 48605 , with the value of ideal MHD growth rate $\gamma_{I}$ larger than $\omega_{* \mathrm{i}} / 2$, the solution is $\omega=\omega_{*_{\mathrm{i}}} / 2+i \sqrt{\gamma_{I}^{2}-\omega^{2}{ }_{*_{\mathrm{i}}} / 4}+\Delta$, with perturbation $\frac{\Delta}{\omega_{A}}=\frac{-i \gamma_{I} \delta \hat{W}_{k}}{\sqrt{\gamma_{I}{ }^{2}-\omega^{2}{ }_{*_{\mathrm{i}}} / 4}}$. The positive imaginary part of $\delta \hat{W}_{k}$ increases the mode frequency, the positive real part of $\delta \hat{W}_{k}$ is stabilizing. For the $\gamma_{I}<\omega_{*_{\mathrm{i}}} / 2$ case, the solution is $\omega=\omega_{*_{\mathrm{i}}} / 2 \pm i \sqrt{\omega_{*_{\mathrm{i}}}^{2} / 4-\gamma_{I}^{2}}+\Delta$, with perturbation $\frac{\Delta}{\omega_{A}}= \pm \frac{\gamma_{I} \delta \hat{W}_{k}}{\sqrt{\omega_{*_{\mathrm{i}}}^{2} / 4-\gamma_{I}^{2}}}$.

With the lowest order radial displacement for $n=1$, the $m=1$ internal kink mode in cylindrical coordinates is $\vec{\xi}_{0}=\xi(r)(\widehat{r}+i \widehat{\theta}) e^{i(\theta-\phi)}$ for $r<r_{s}$, zero for $r>r_{s}$, and the non-resonant part of kinetic contribution $\delta W_{n}=-\int\left(\vec{\xi}_{\perp} \cdot \nabla \mathrm{p}\right)\left(\vec{\xi}_{\perp} \cdot \vec{\kappa}\right) \mathrm{d} \overrightarrow{\mathrm{r}}$ can be reduced to $\delta \hat{W}_{n}=\frac{2^{7 / 2} \pi}{r_{s}^{2}} \sum_{\sigma} \int \frac{E^{3 / 2} \cos (\theta)}{B} \partial_{r} F r \mathrm{~d} r \mathrm{~d} \theta \mathrm{d} \lambda \mathrm{d} E$, with the fast ion distribution uniform in poloidal angle and pitch $\lambda=\sqrt{1-\mu B / E}$. Normally the fast ion density gradient $\partial_{r} F$ is negative for the slowing down distribution which is peaked in the core region. So fast ions deeply trapped in the bad curvature region will be destabilizing with negative $\cos (\theta)$, passing or near passing-trapped boundary fast ions will be stabilizing by increasing the fast ion pressure in the good curvature region and positive $\cos (\theta)$.

For cases of fast ion pressure comparable to the background plasma's, it is instructive to obtain the analytical solutions of the dispersion relation with a fast ion distribution model. Consider a slowing down distribution of deeply trapped beam ions, the deposition function can be taken as $F(E, r, \mu)=E^{-3 / 2} f(r) \delta(\mu-\alpha B)$, with $E_{0}<E<E_{m}$. After simplification, the ideal limit of dispersion function but keeping 
diamagnetic effect can be reduced to $-i \sqrt{\omega\left(\omega-\omega_{* \mathrm{i}}\right)}-\gamma_{I}+\frac{\beta_{h} \omega}{\pi \beta_{c}} \ln \left(\frac{\omega-\omega_{d m}}{\omega-\omega_{d 0}}\right)=0$, with critical value $\beta_{c}=\frac{4 r_{s}^{2} \bar{\omega}_{d}}{\pi c_{f} a R \omega_{A}}$ and normalized value $\beta_{h}=\frac{8 \pi}{B_{0}^{2} V} \int E d N$ [6]. To find the threshold value with this dispersion function for which the frequency $\omega=\omega_{r}+i 0$ is real, the real and imaginary part of dispersion function give $\pi \gamma_{I}=\frac{\beta_{h} \omega}{\pi \beta_{c}} \ln \left(\frac{\omega_{d m}-\omega}{\omega-\omega_{d 0}}\right)$ and $\sqrt{\omega\left(\omega-\omega_{* \mathrm{i}}\right)}=\frac{\beta_{h} \omega}{\beta_{c}}$. Similar to perturbative analysis, the behavior of solution depends on the discriminant $\gamma_{I}^{2}-\omega_{*_{\mathrm{i}}}^{2} / 4$. In case $\gamma_{I}<\omega_{*_{\mathrm{i}}} / 2$ with $\beta_{h}=0$, the solution $\omega=\omega_{*_{\mathrm{i}}} / 2 \pm i \sqrt{\omega_{*_{\mathrm{i}}}^{2} / 4-\gamma_{I}^{2}}$ have two marginal branches at interval $\left[0, \omega_{*_{i}}\right]$. When increasing fast ion beta $\beta_{h}$, the higher frequency branch will be destabilized first and then stabilized further with real frequency around $\omega_{*_{i}}$, and it is suggested to be the cause of diamagnetic fishbone. However, this diamagnetic fishbone branch can exist even without fast ions.

In case $\gamma_{I}>\omega_{*_{\mathrm{i}}} / 2$, there are also two mode branches for this dispersion relation, one is the MHD branch, which is confirmed to be the sawtooth, unstable when the ideal fluid potential energy contribution $\delta W_{M H D}$ is negative at high plasma beta $\beta_{T}$. This ideal kink branch is a low fast ion $\beta_{h}$ and low frequency phenomenon. The other branch is the precessional fishbone, a high fast ion $\beta_{h}$ and high frequency instability. At $\beta_{h}=0$, the sawtooth branch has a finite growth rate which depending on $\delta W_{M H D}$ and can be stabilized with increased $\beta_{h}$. The fishbone growth rate is $\omega=-i \infty$ for $\beta_{h}=0$ and has a threshold fast ion $\beta_{h}$ for destabilization [11, 12].

Details of these solutions can be found in Ref. 6 and we are interested in the stable regime for sawtooth and fishbone in the case of $\gamma_{I}>\omega_{*_{\mathrm{i}}} / 2$. With the ideal limit of dispersion function and fast ion distribution model, the existence conditions for the stable regime was summarized as (a) the ideal mode growth rate is limited by Eq. 
6.125 in Ref. 6, which mean the plasma is strongly unstable, (b) the range of fast ion beta $\beta_{h}$ is given approximately by Eq. 6.126 in Ref. 6, (c) the plasma should not be very far away from the ideal limit which means the resistivity effect is small, (d) fast ion's energy is sufficiently high. It was worth to note that there are a lot of fast ion distributions which can give this stable gap, both from auxiliary heating and fusion reaction. In general, the fast ion's energy from high power ICRH is higher than NBI at present tokamak, which can stabilize sawtooth more effectively.

With the reduced ideal limit dispersion relation, we evaluated these two thresholds of the stable regime by scanning the real frequency from $\omega_{*_{\mathrm{i}}}$ to $\omega_{d m}$ in EAST fishbone shot and obtained two threshold values. $\omega_{d m}$ is the maximum toroidal precession frequency, $\bar{\omega}_{d}$ in $\beta_{c}$ is the particle averaged value, $c_{f}=\sqrt{\pi} a / h=\sqrt{\pi} a / 0.466$ is a shape constant for the fast ion density Gaussian profile $f(r) \sim \mathrm{e}^{-(r / h)^{2}}, \quad$ as is shown in fig 6. The sawtooth stabilization threshold is $\beta_{\text {thresh }-s t}=2.64 * 10^{-3}$, the mode real frequency is $\omega_{s t} / \omega_{0}=3.01 * 10^{-4}$ with $\omega_{0}=8.86 * 10^{7} \mathrm{rad} / \mathrm{s}$ the cyclotron frequency, or $f_{s t}=4.24 \mathrm{kHz}$. The fishbone destabilization threshold is $\beta_{\text {thresh }-f b}=4.75 * 10^{-3}$, with real frequency $f_{f b}=5.86 \mathrm{kHz}$. This analytical solution based on the reduced model indicates a stable domain for sawtooth and fishbone with fishbone shot in EAST, and gives a reference for the numerical solution. With general fast ion distribution and realistic plasma equilibrium, the kinetic term should be evaluated numerically. 


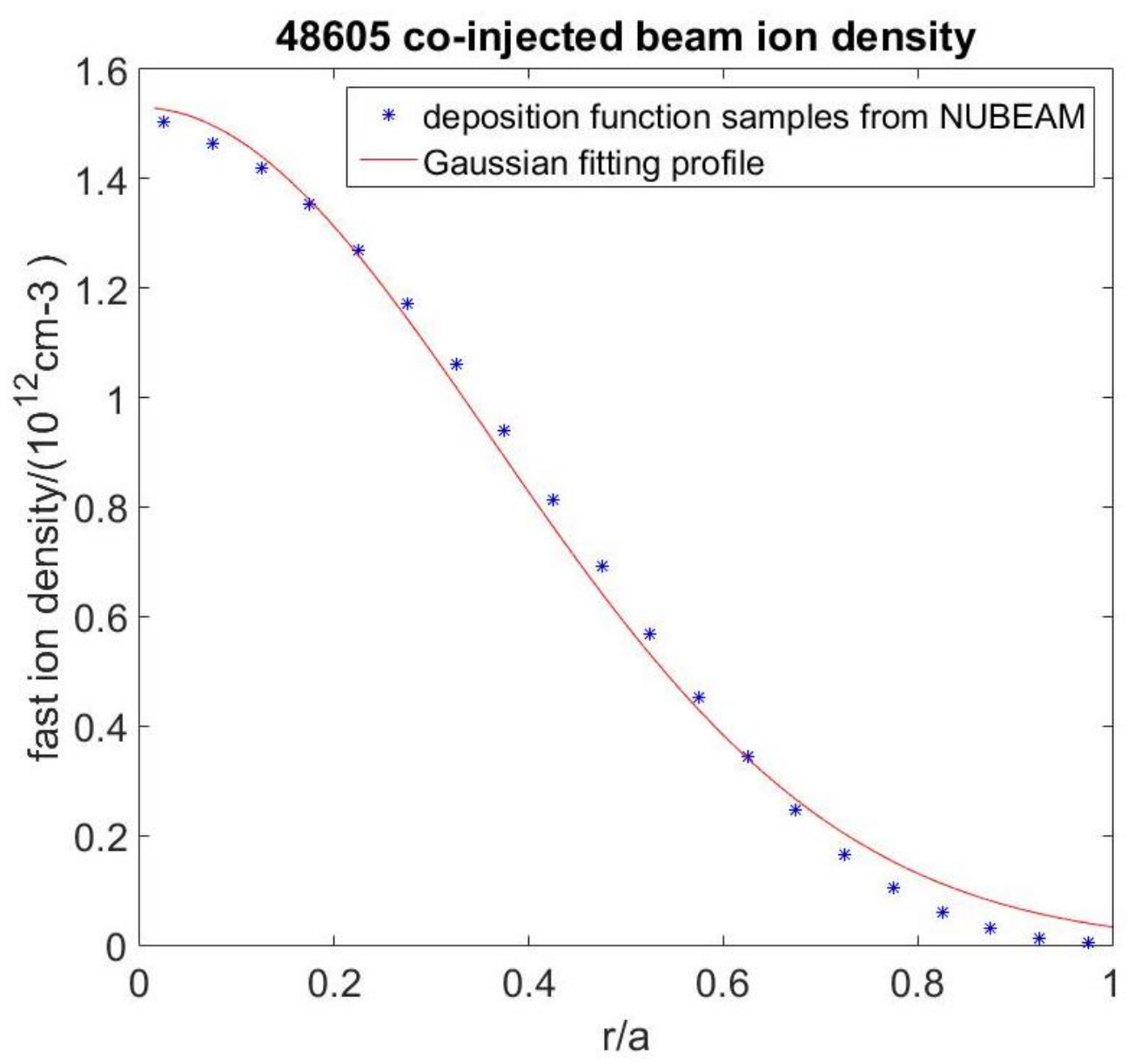

Figure 6. Fast ion density profile fitted with Gaussian function for shot 48605 .

\section{Numerical results with co and counter beam injection}

With a sample fast ion distribution in a realistic plasma equilibrium, the kinetic contribution in the fishbone dispersion relation is evaluated numerically in this section. It is a general method developed in the Hamiltonian guiding center code ORBIT using the fast ion distribution from EAST, which considers finite orbit width, plasma shape and finite beta. The kinetic contribution is expressed as $\delta \widehat{W}_{k}=\frac{-V \beta_{h}}{4 \pi^{2}\left(r_{\mathrm{s}} \xi\right)^{2} \sum_{j} E_{j}} \sum_{k}\left[\frac{\partial_{\Psi_{p}} F}{F} E H-\sum_{l} \frac{4 E^{2} Q}{F} \frac{\bar{J}^{*} \bar{J}}{\omega_{d}-\omega+(n q-l) \Omega_{\mathrm{t}}}\right]_{\mathrm{k}}$ after bounce orbit averaged, which in Monte Carlo particle representation is a sum over particles in the distribution $\sum_{k}$. The sum $\sum_{l}$ is over resonances $l$ from -3 to 4 neglecting higher order terms. The denominator in the resonance contribution is the bounce precession resonance condition between fast ions and a coherent mode. For the fishbone mode, the $n=1, m=1$ perturbation dominates. $q-l=0$ was the first observed fishbone in PDX with deeply trapped particle precession frequency, 
$q-l= \pm 1$ dominates the mode particle resonance for a large bounce angle trapped particle and passing particle population.

The kinetic contribution is determined by the equilibrium, perturbed magnetic field and fast ion distribution. The safety factor profile and plasma displacement of the fishbone are shown in fig 5, which is primarily an $n=1, m=1-3$ mode. The code has included mode particle interaction for high poloidal harmonics in $H\left(\Psi_{\mathrm{p}}, \theta\right)=\left(\vec{\xi}_{\perp} \cdot \vec{\kappa}\right)\left(\vec{\xi}_{\perp} \cdot \nabla \Psi_{\mathrm{p}}\right) \quad$ and $\mathrm{J}\left(\Psi_{\mathrm{p}}, \theta\right)=(1-\mu B / E)\left(\vec{\xi}_{\perp} \cdot \vec{\kappa}\right)$. The classical slowing down of a co injected beam ion distribution is shown in fig 3, the corresponding bounce averaged toroidal precession frequency and poloidal transit frequency are shown in fig 7, fig 8 and fig 9 . As is shown in fig 7, the trapped particle distribution has a well-defined precession frequency located at $0-10 \mathrm{kHz}$, the toroidal precession frequency of co passing fast ions has a wide spread distribution up to 200 $\mathrm{kHz}$. This slowing down distribution has assembled of fast ion which characteristic frequency comparable to $n=1$ MHD mode frequency and much higher than bulk ion diamagnetic frequency at $\mathrm{q}=1$ surface.
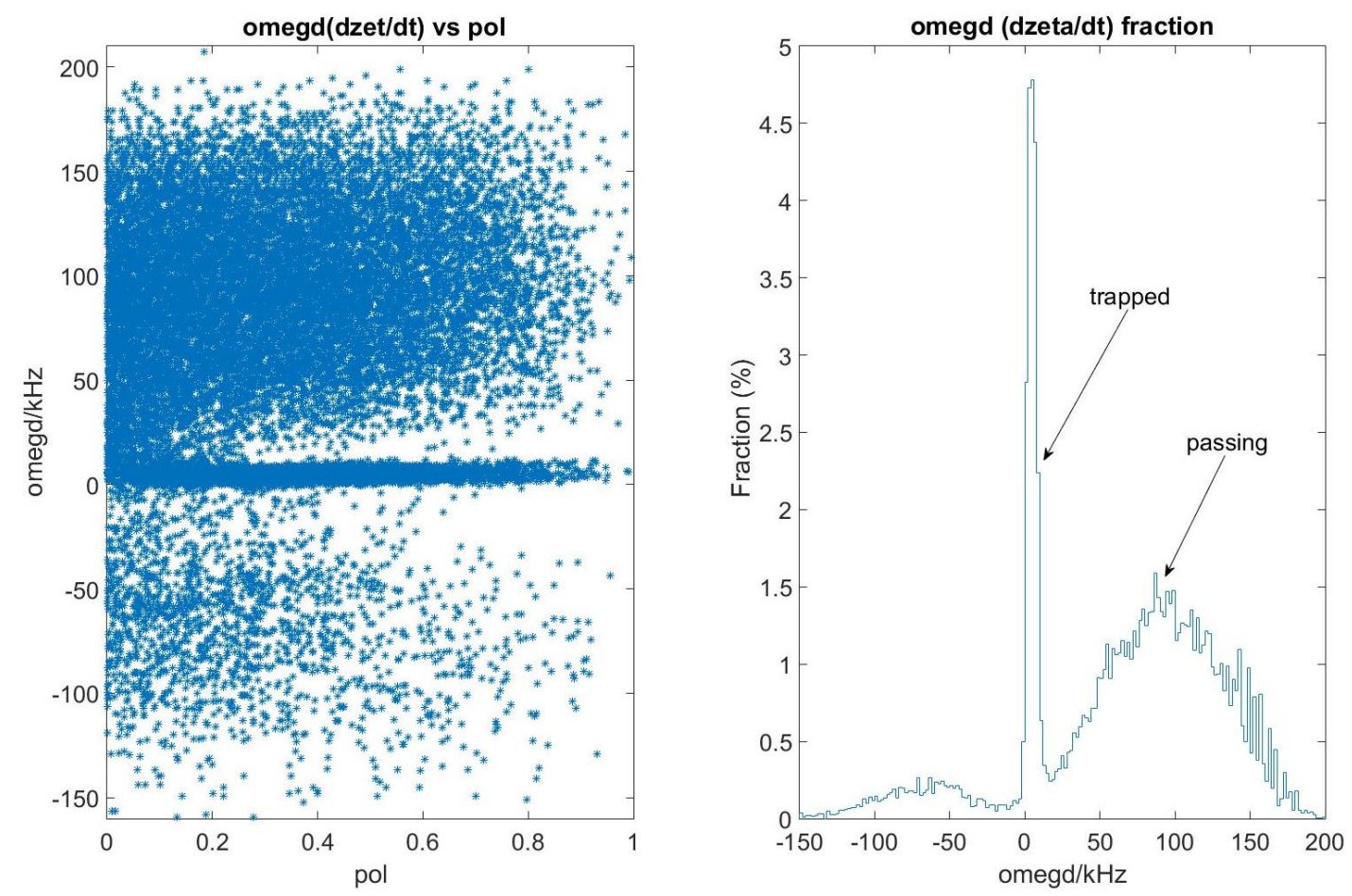

Figure 7. Co injected beam ion's toroidal precession frequency distributions for shot 48605. 

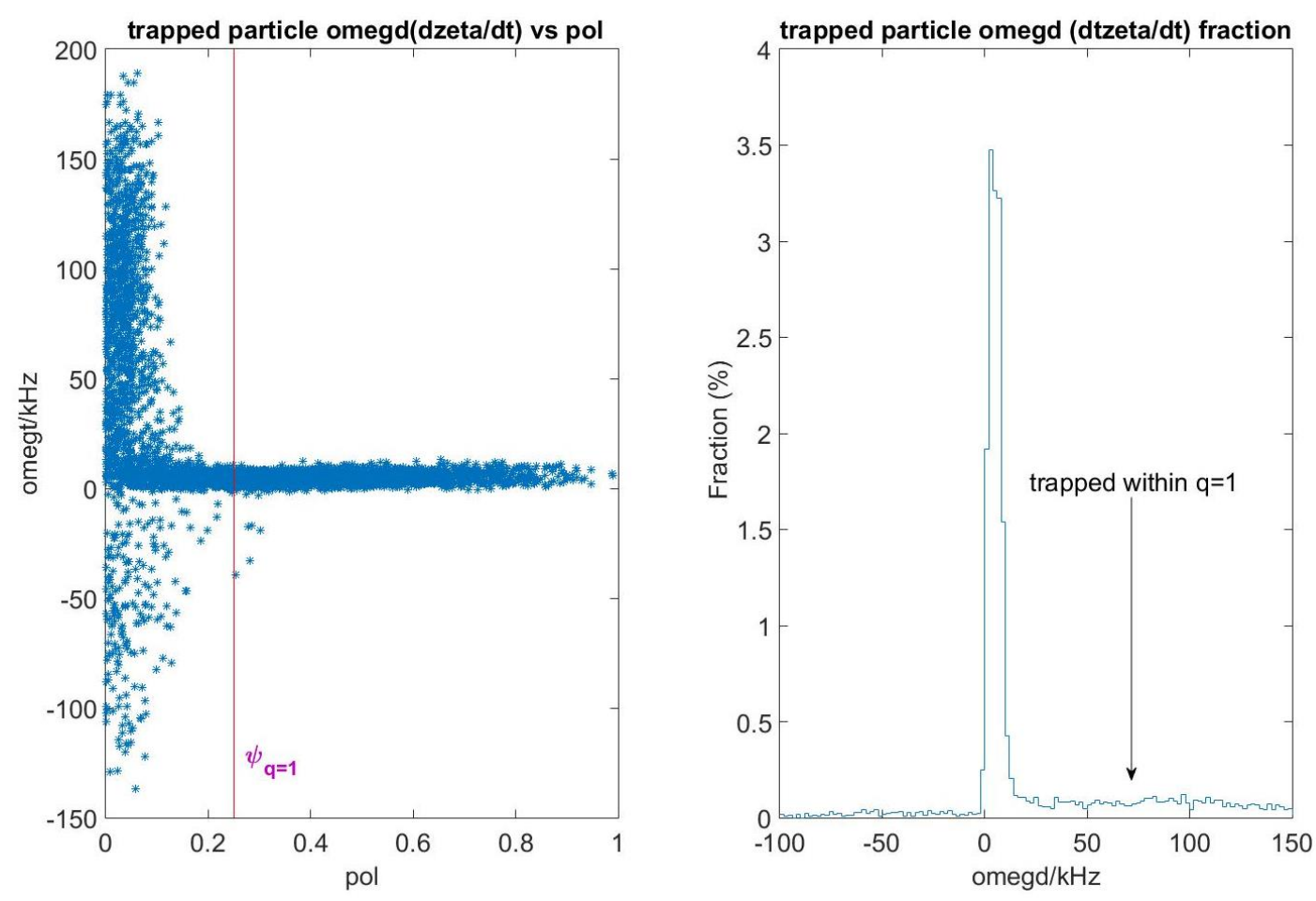

Figure 8. Trapped beam ion' toroidal precession frequency distributions for shot 48605.
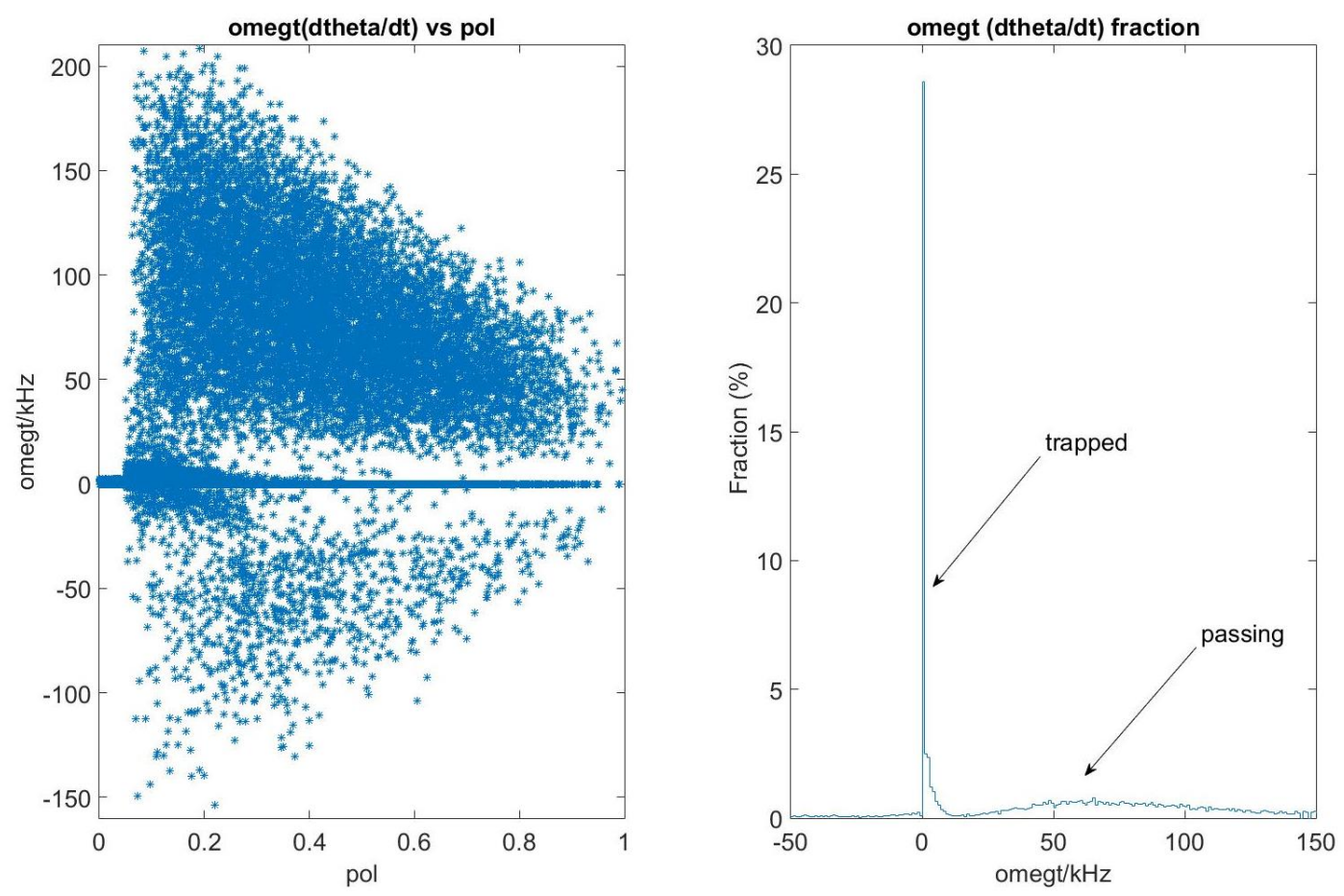

Figure 9. Co injected beam ion's poloidal transit frequency distributions for shot 48605.

As is shown in section 3, the analytical solution of the general fishbone dispersion relation has two solutions, sawtooth and fishbone. To compare numerical solutions 
with experiment data, a root finding procedure have been developed in ORBIT which can carry out integrals over a Monte Carlo fast ion distribution and evaluate bounce averaged quantities. With initial solution $\omega=\omega_{*_{\mathrm{i}}} / 2+i \sqrt{\gamma_{I}^{2}-\omega_{*_{\mathrm{i}}}^{2} / 4}+\Delta \quad$ in $\gamma_{I}>\omega_{*_{\mathrm{i}}} / 2$ perturbation analysis, we increased the fast ion $\beta_{h}$ and tracked the solutions. The dispersion solutions of the sawtooth branch are shown in fig 10, with a marginal unstable sawtooth mode when $\beta_{h}$ zero, and strongly stabilized with increased fast ion density, its real frequency located at around the initial value $\omega_{*_{\mathrm{i}}} / 2$. The EAST beam heating experiment observed the sawtooth period increased by co-current beam injection. Sawtooth research reveals this periodic relaxation is not a pure MHD instability, which can be influenced by fast ion distributions, sheared toroidal flows, diamagnetic drift effect and some other mechanisms. This work only focuses on the energetic particle effect [13].

Sawtooth stabilization by trapped particles originates from the conservation of the third adiabatic invariant, which is equal to poloidal magnetic flux enclosed by the drift orbit. It acts as a current loop contraction and extracts energy from the fast ion pressure gradient, requiring that the trapped particle drifts across the field line rapidly, with the precession frequency much larger than the perturbation frequency. As shown in fig 7 and fig 9, the real frequency of the sawtooth solution is much smaller than the fast ion characteristic drift frequency. The trapped fraction is about $27 \%$ in this fast ion distribution, the precession frequency distribution is shown in fig 8 , the effect from trapped particles and from passing particles is competitive.

For the passing particle effect, first consider the non-resonant part of the kinetic contribution $\delta W_{n}=-\int_{0}^{r_{s}}\left(\vec{\xi}_{\perp} \cdot \nabla \mathrm{p}\right)\left(\vec{\xi}_{\perp} \cdot \vec{\kappa}\right) \mathrm{d} \overrightarrow{\mathrm{r}}$, the kink perturbation primarily within $\mathrm{q}=1$ surface, fast ion pressure gradient $\nabla p<0$ is negative with on-axis beam injection in EAST. As is shown in fig 7-9, particles within the $\mathrm{q}=1$ surface only constitute a small fraction of total fast ion population. The $\mathrm{q}=1$ surface is located at around $\psi_{p}=0.25$, and these passing particles cancel contributions between the good curvature and adverse curvature regions, they do not influence the sawtooth instability. Other co passing particles launched from outstide the $q=1$ surface in the adverse curvature region will cross the $\mathrm{q}=1$ surface in the good curvature region and stabilize the sawtooth. Beam injection deposition mainly occurs in the adverse curvature region as is shown in fig 1. Particles near the trapped-passing boundary still have a significant stabilization effect, with orbit width comparable to the perturbation radial extent, increasing the fast ion pressure in the good curvature region. Intuitively, counter beam injection will destabilise the sawtooth in EAST, the final effect requires an examination of the equilibrium and fast ion distribution in whole, especially the local gradient near the $\mathrm{q}=1$ surface [14]. 

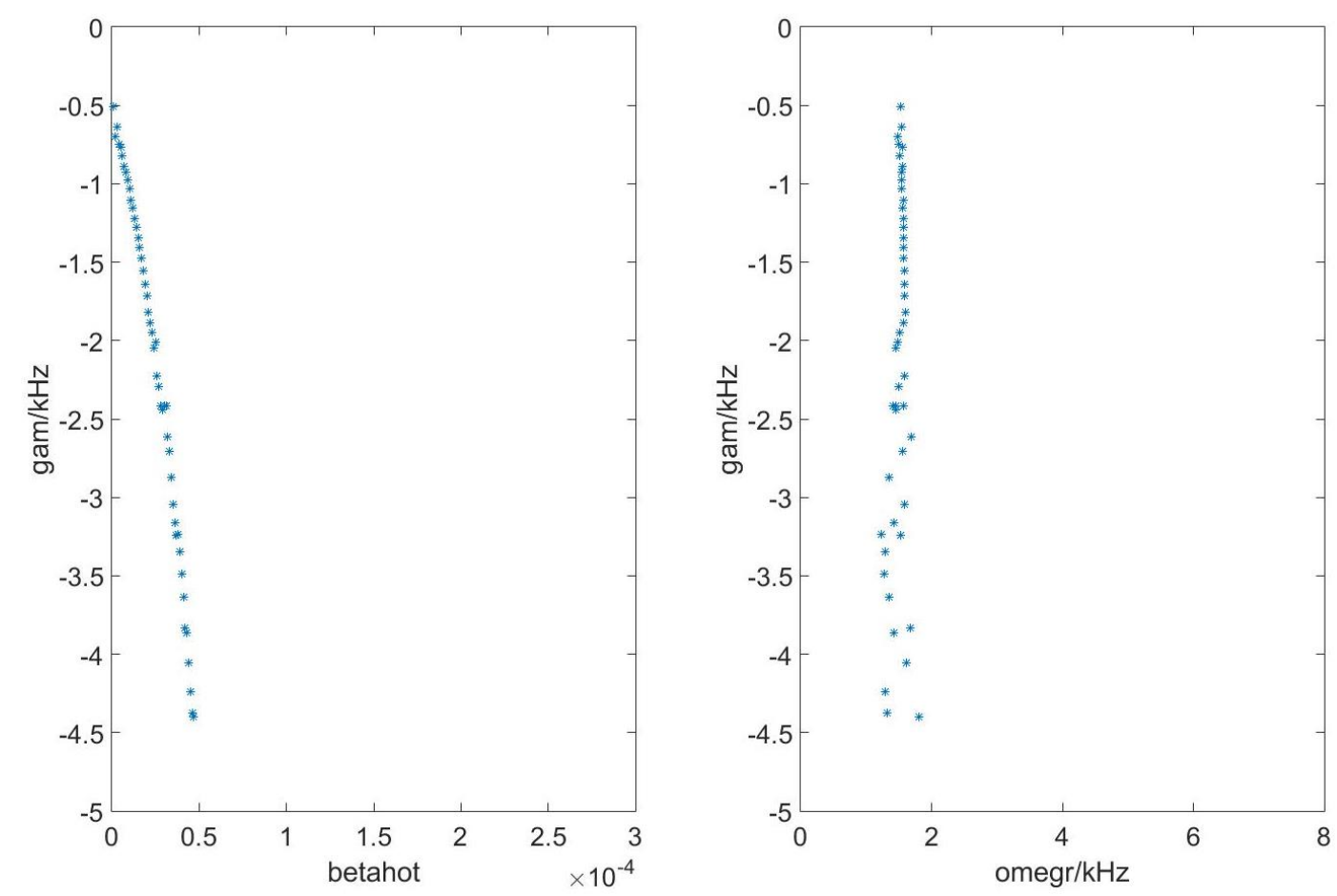

Figure 10. Growth rate of sawtooth dispersion solutions as a function of fast ion $\beta_{h}$ and its complex plane.
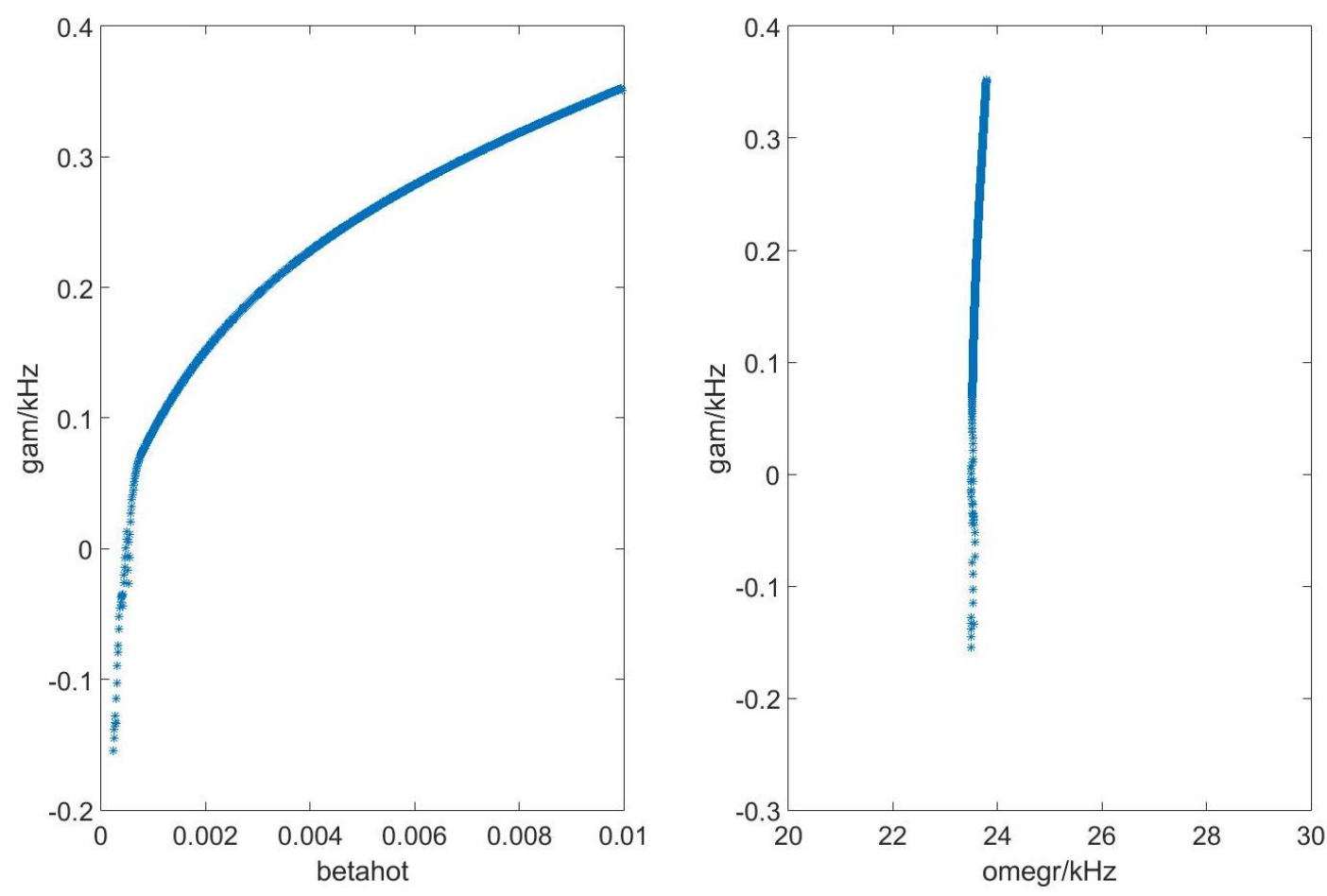

Figure 11. Growth rate of fishbone dispersion solutions as a function of fast ion $\beta_{h}$ and its complex plane. 

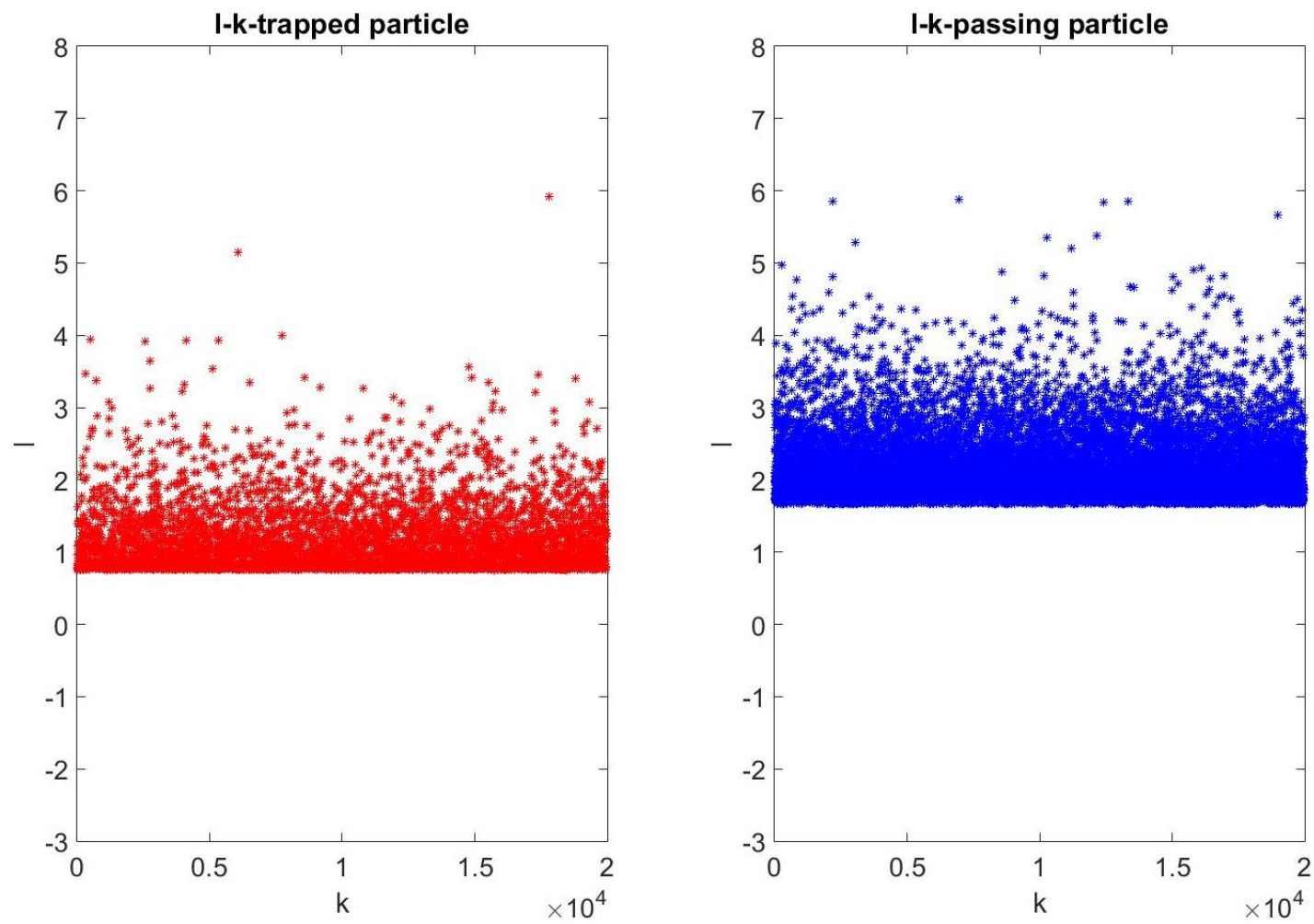

Figure 12. Resonance order distribution for trapped and passing particles.

This precessional fishbone branch is a high fast ion $\beta_{h}$ forced oscillations, the work tracks the fishbone solution step by step decreasing from an initial value of $\beta_{h}=10 \times 10^{-3}$. First, search the best initial complex value of dispersion function within a wide enough frequency range according to characteristic frequency distribution shown in fig 7 and fig 9. Fishbone solution is shown in fig 11 with fast ion $\beta_{h}$ decreasing from $1 \times 10^{-2}$ to 0 . The fishbone real frequency approaches minus infinite when the beam ion beta $\beta_{h}$ decreases to zero, as is shown in complex frequency plane. The fishbone destabilization threshold $\beta_{h}$ under co-beam injection is $4.7 \times 10^{-4}$, the real frequency is about $24 \mathrm{kHz}$, which is close to the measurement. The calculation gives a stable regime $0-4.7 \times 10^{-4}$ of beam ion $\beta_{h}$ for stabilization of sawtooth and fishbone.

With the fishbone dispersion solution and resonance condition $\omega=\omega_{d}+(n q-l) \Omega_{\mathrm{t}}$, the work examined the resonance condition between mode and fast ions, which strongly depends on equilibrium, perturbation field and fast ion distribution. The 
distribution of poloidal resonance order $q-l$ with total 20000 particles is shown in fig12, deeply trapped particles have a strong resonance at the precession frequency $\omega \sim \omega_{d}$, the dominant resonant frequency of passing particles is about $\omega \sim \omega_{d}-\Omega_{t}$. Both trapped particles and passing particles can contribute to fishbone destabilization. In threshold evaluation of dispersion function in EAST beam experiment analysis, we also explored the stable domain under different beam injection scenarios. Fig 13 shows the beam ion distribution under co current injection and counter current injection with the same beam parameters. The retention of co-beam ions is higher than counter beams. Fig 14 shows the precession frequency distribution, with the counter precession frequency half of co current. The dispersion solution for this fast ion classical slowing down distribution is shown in fig 15 and fig 16. A marginal unstable kinetic kink mode is further stabilized with increasing beam ion's $\beta_{h}$. Fishbone threshold is about $6.5 \times 10^{-4}$, real frequency is about $25 \mathrm{kHz}$.

The stable window with balanced beam injection scenario for both sawtooth and fishbone is wider than co current injection. In order to optimize the beam heating power in exploration of long pulse high beta regime in EAST based on the present NBI layout, the result suggests a beam heating scenario with a modulated beam pulse injection with synchronization of the co and counter beams, between the adjacent beam pulses, the relative small amplitude sawtooth was allowed to crash deliberately. The single beam pulse turn on time should be shorter than plasma energy confinement time and longer than beam ion slowing down time, for sake of avoiding monster sawtooth and heating plasma. For the fishbone shot this paper analyzed, the plasma energy confinement time is about $150 \mathrm{~ms}$, the beam ion slowing time is about $50 \mathrm{~ms}$. Sawtooth period longer than plasma energy confinement time has the potential to grown to a monster sawtooth. In addition to obtain higher plasma beta in this stable domain, the modulated beam injection scenario can prolong the NBI duty time significantly. For direct current beam injection, the duty time of EAST NBI is the order of $10 \mathrm{~s}$, and modulated injection can be the order of $100 \mathrm{~s}$, such as the $96 \mathrm{~s}$ beam heating shot 59814 in EAST [15]. The next goal for EAST is demonstrate $400 \mathrm{~s}$ H-mode with high performance. Details of this scenario in EAST need further investigation for other complicate sawtooth and fishbone physics [13, 14]. 


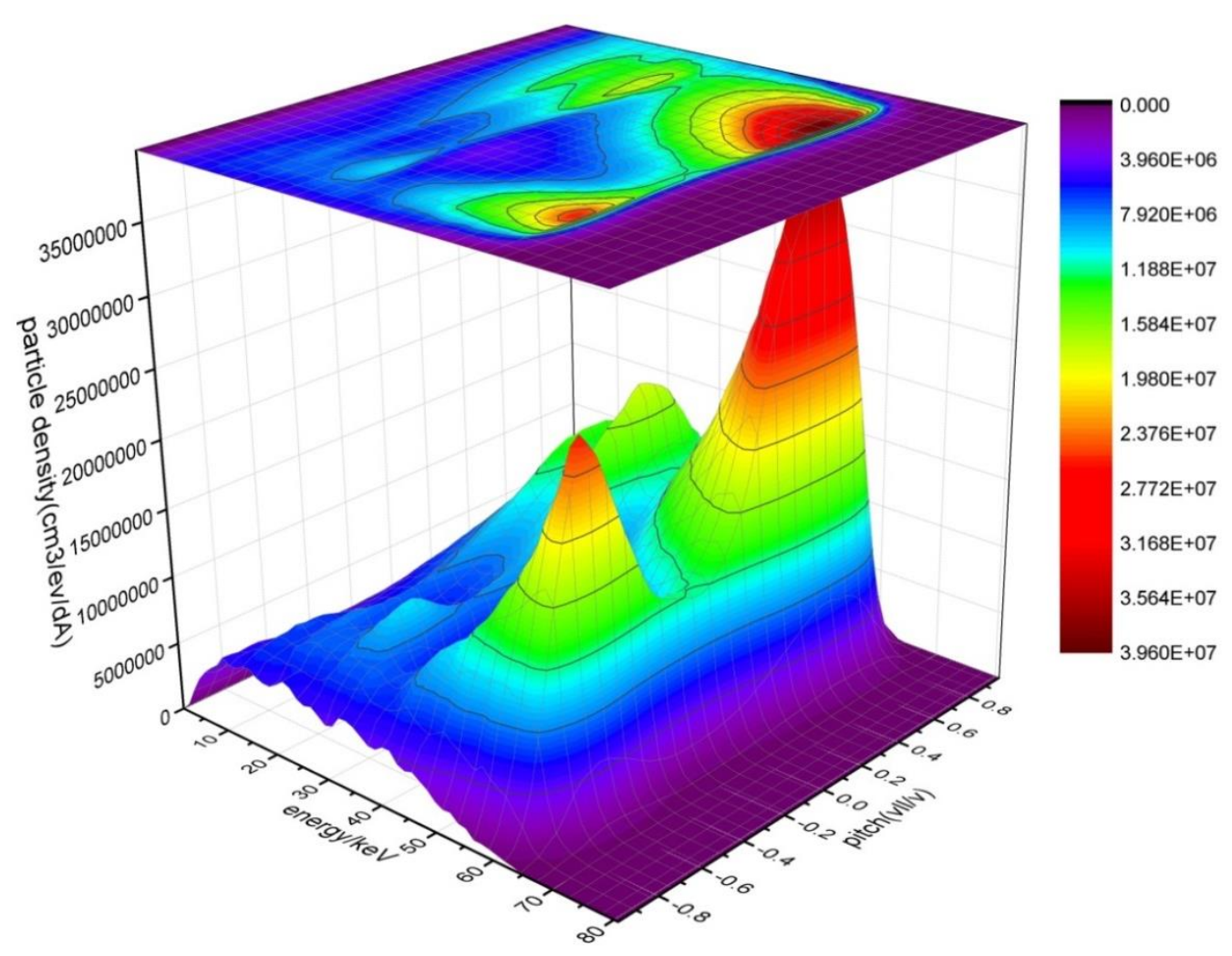

Figure 13. Beam ion's pitch and energy distribution from four beam injectors with the same beam parameters.
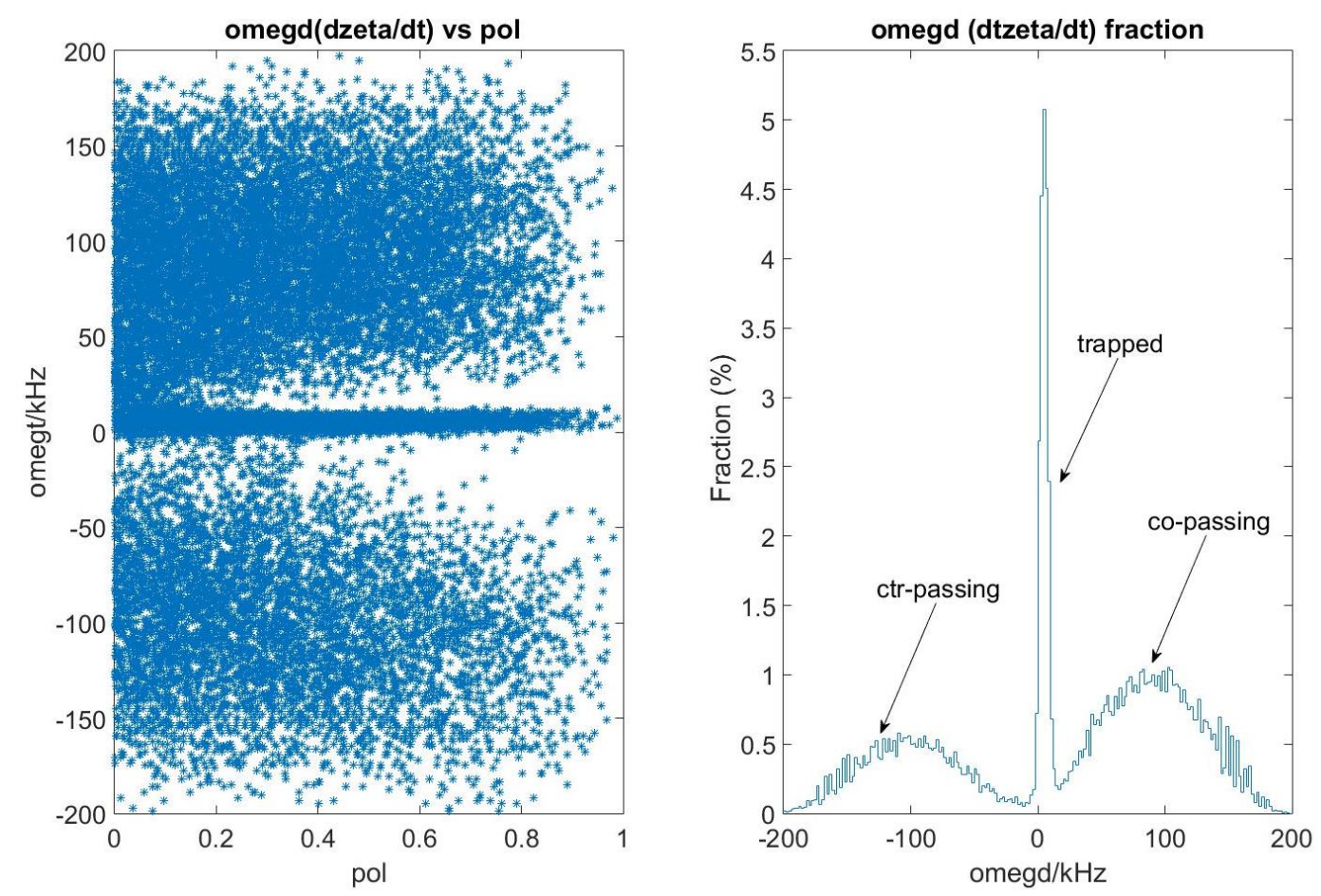

Figure 14. Fast ion's toroidal precession frequency of classical slowing down distribution from four beam injectors. 

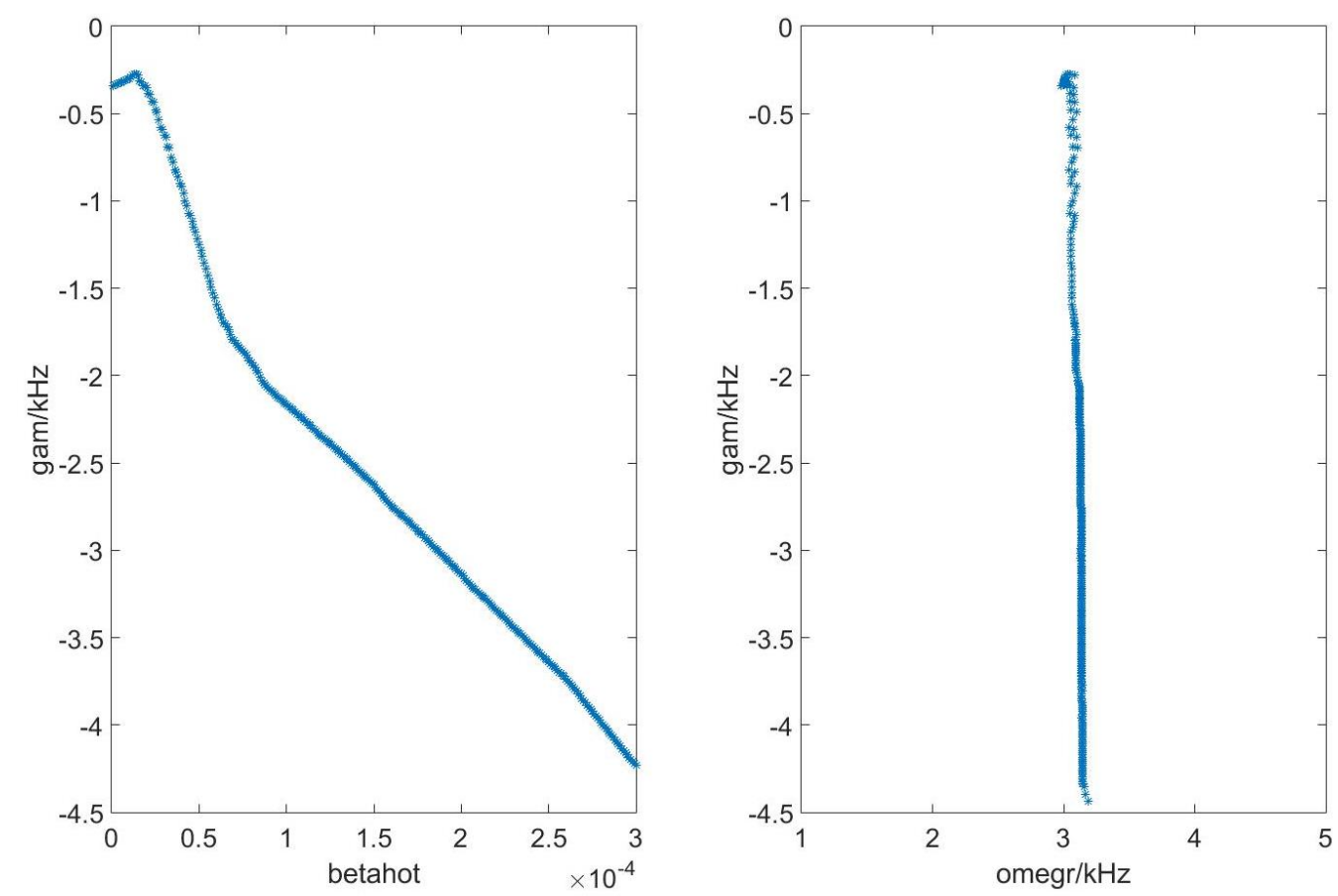

Figure 15. Growth rate of sawtooth dispersion solutions as a function of fast ion $\beta_{h}$ and its complex plane under balance beam injection.
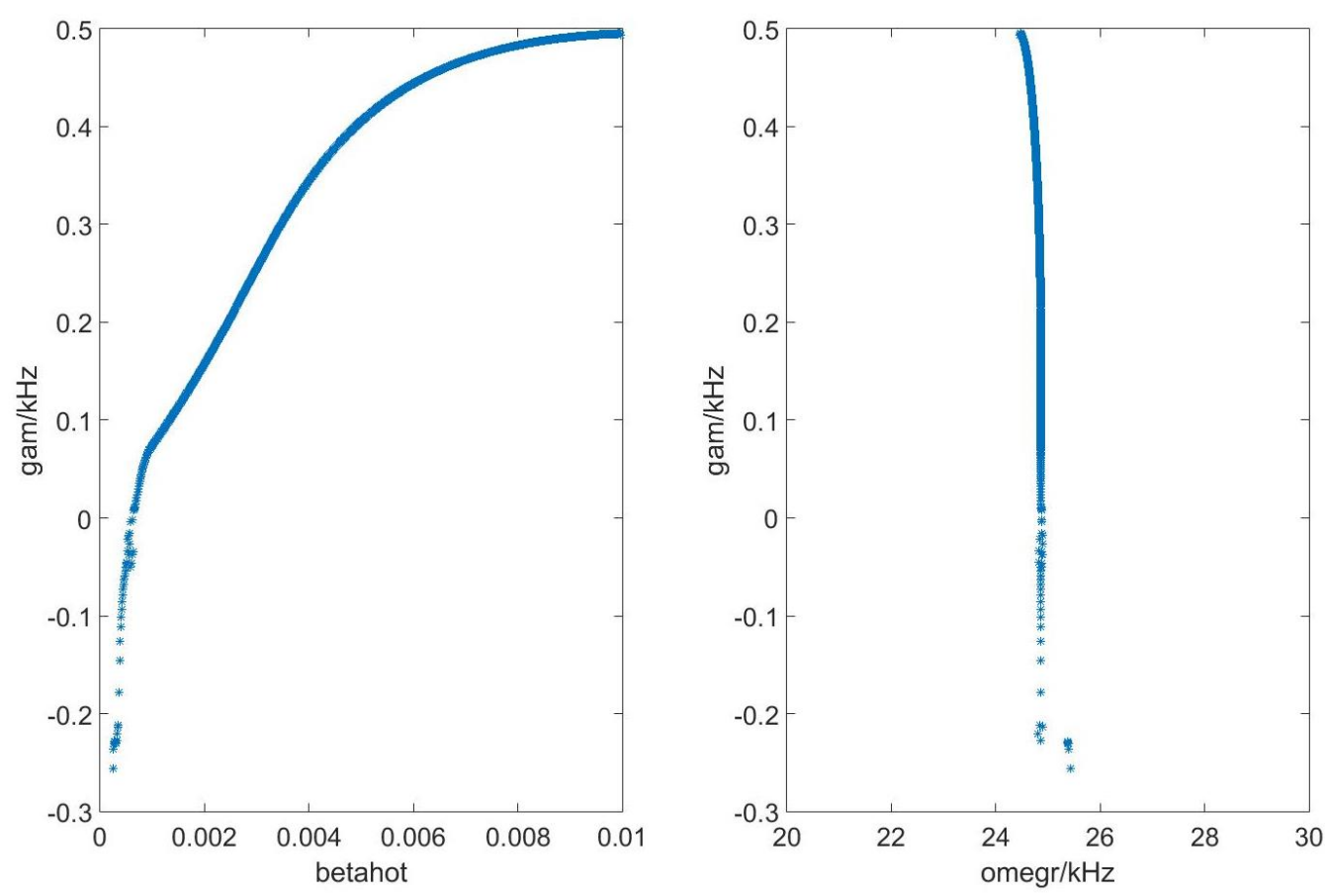

Figure 16. Growth rate of fishbone dispersion solutions as a function of fast ion $\beta_{h}$ and its complex plane under balance beam injection.

\section{Fast ion loss induced by fishbone perturbation}

The work investigated the fast ion loss with guiding center following code ORBIT 
in Boozer coordinates, the equilibrium is produced by the code EFIT, with the eigenfunction modeled with the ideal stability code NOVA. The perturbation amplitude and frequency have been scaled with experiment, the evolution of maximum radial displacement diagnosed by high resolution soft $X$ rays is shown in fig 17, the absolute maximum amplitude is about $1.5 \mathrm{~cm}$, the mode structure measured by SX-system tomography indicates that the structure is maintaining a top-hat shape. The frequency spectrogram is in a broad range 15-20 kHz. During the fast ion loss calculation in one fishbone burst of about $2 \mathrm{~ms}$, the perturbation amplitude and frequency were kept constant for simplicity. With maximum perturbation value and $20 \mathrm{kHz}$ frequency, the calculation shows about $15 \%$ particle loss after one fishbone burst time [16].

As is shown in fig 4, the drop in neutron rate is about $6.7 \%$. If one neglects the neutron drop due to the fast ion redistribution, the fast ion loss fraction can be estimated from the neutron rate evolution. A TRANSP calculation with this fishbone shot plasma parameters indicates $93.7 \%$ fusion neutrons from beam-target reaction, $6.3 \%$ from beam-beam reaction. Neutron rate drop is $\delta S / S=0.937 * 2 * \frac{\delta n_{f i}}{n_{f i}}+0.063 * \frac{\delta n_{f i}}{n_{f i}}, \delta n_{f i} / n_{f i}$ is fast ion loss fraction, which deduced to $\delta n_{f i} / n_{f i}=6.3 \%$. The difference is due to the fixed mode amplitude and ignorance of the neutron contribution, a detailed calculation with a more accurate model needs further work [17].

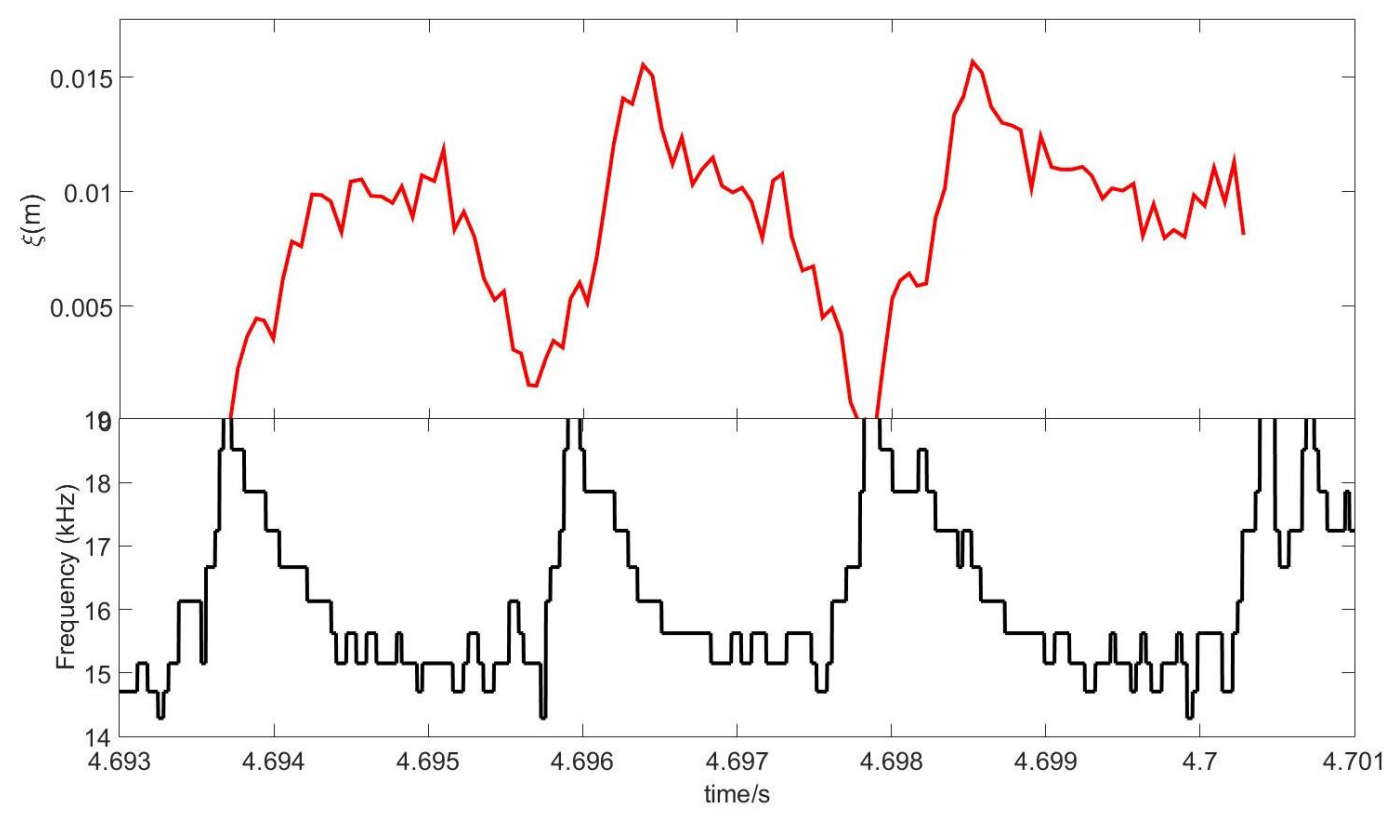

Figure 17. Time evolution of absolute fishbone perturbation amplitude and instability spectrogram obtained from Mirnov and SX signals.

\section{Conclusion and discussion}


This paper investigated the stabilizing-destabilizing property with fishbone shot parameters in EAST. The dispersion relation produced two solutions both analytically and numerically in the work, the kinetic kink mode and fishbone. We assumed that the coupled system didn't vary significantly above threshold, which assumes that the mode amplitude growth didn't change the equilibrium and fast ion distribution. We used a fixed fast ion distribution obtained by TRANSP/NUBEAM classical slowing down calculation, ideal fluid drive and eigenfunction calculated by stability code NOVA. The perturbation in the model kept small and fixed, through changing the fast ion beta with a fixed distribution, we obtain a marginal unstable sawtooth solution near ideal MHD threshold, further stabilized with increased fast ion $\beta_{h}$ both during co-beam injection and balanced injection. Fishbone destabilization has a threshold in fast ion $\beta_{h}$, with characteristic frequency determined by resonant fast ions both from the trapped and passing populations. The model can't reproduce the fishbone frequency chirping which need a self-consistent nonlinear time dependent model. The results indicated the fishbone mode in this shot is a high frequency branch which tapping energy from gradient of resonant fast ion distribution, the theory mainly developed by Chen, White and Rosenbluth [9].

The balance beam injection in EAST has a wider stable regime for sawtooth and fishbone than co-beam injection itself, the suggestion is to modulate all four beam injectors in step with the single pulse period smaller than plasma confinement time and longer than beam ion slowing down time, benefiting long pulse high beta plasma discharge. It is worth noting that even outside the stable domain, fishbone perturbation has the similar effect of sawtooth on bulk plasma, such as core plasma profile flattening and impurity ejection. It has been found that the more beam power, the more fishbone frequent in EAST.

For burning plasma in fusion reactor, due to larger machine size and stronger magnetic field, the characteristic drift frequency of alpha particle is similar to the beam injected fast ion's in present facility, the same stable window can be obtained by fusion alphas in burning plasma and a quantitative investigation needs more work [11, 12]. The sawtooth period is expected to be increased by large amount of alpha particle, the sawtooth control actuators have been considered the ECCD, ICRF and NBI [20].

\section{Acknowledgements}

The authors wish to acknowledge conversations with Nikolai Gorelenkov and Marina Gorelenkova. We are also grateful for the support of the EAST Contributors. Work supported by the National Nature Science Foundation of China with contract Nos. 11675211 and 11875290.

\section{References}

[1] N. N. Gorelenkov, S. D. Pinches and K. Toi 2014 Energetic particle physics in fusion research in preparation for burning plasma experiments. Nucl. Fusion, 54(11), 125001. 
[2] R. B. White, P. H. Rutherford, P. Colestock, M. N. Bussac 1988 Sawtooth Stabilization by Energetic Trapped Particles. Phys. Rev. Lett. 60(20), 2038.

[3] W W Heidbrink, M E Austin, R K Fisher, M Garcia-Munoz, G Matsunaga, G R McKee, R A Moyer, C M Muscatello, M Okabayashi, D C Pace, K Shinohara, W M Solomon, E J Strait, M A Van Zeeland and Y B Zhu 2011 Characterization of off-axis fishbones. Plasma Phys. Control. Fusion 53 (8), 085028.

[4] Wei Shen, Feng Wang, G.Y. Fu, Liqing Xu, Guoqiang Li and Chengyue Liu 2017 Hybrid simulation of fishbone instabilities in the EAST tokamak. Nucl. Fusion, 57(11), 116035.

[5] Youbin Pei, Nong Xiang, Youjun Hu, Y. Todo, Guoqiang Li, Wei Shen, and Liqing Xu 2017 Kinetic-MHD hybrid simulation of fishbone modes excited by fast ions on the experimental advanced superconducting tokamak (EAST). Phys. Plasmas, 24(3), 032507.

[6] R. B. White 2014 The theory of toroidally confined plasmas. 3th ed. Singapore: World scientific publishing company, p 259.

[7] Liqing $\mathrm{Xu}$, Jizong Zhang, Kaiyun Chen, Liqun Hu, Erzhong Li, Shiyao Lin, Tonghui Shi, Yanmin Duan and Yubao Zhu 2016 Fishbone activity in experimental advanced superconducting tokamak neutral beam injection plasma. Phys. Plasmas 22(12), 122510.

[8] Bin Wu, Baolong Hao, R. B. White, Jinfang Wang, Qing Zang, Xiaofeng Han, and Chundong Hu 2017 Calculation of prompt loss and toroidal field ripple loss under the neutral beam injection on EAST , Plasma Phys. Control. Fusion, 59(2) 025004.

[9] Liu Chen, R. B. White, M. N. Rosenbluth 1984 Excitation of Internal Kink Modes by Trapped Energetic Beam Ions. Phys. Rev. Lett. 52(13), 1122.

[10] R. B. White, L. Chen, F. Romanelli, R. Hay 1985 Trapped particle destabilization of the internal kink mode. Physics of Fluids 28(1), 278. doi: 10.1063/1.865198

[11] R. B. White, F. Romanelli, and M. N. Bussac 1990 Influence of an energetic ion population on tokamak plasma stability. Physics of Fluids B: Plasma Physics 2(4), 745.

[12] Yanlin Wu, C. Z. Cheng, and R. B. White 1994 Alpha particle effects on the internal kink and fishbone modes. Physics of Plasmas 1, 3369.

[13] I. T. Chapman, S. D. Pinches, L. C. Appel, R. J. Hastie, T. C. Hender, S. Saarelma, S. E. Sharapov, and I. Voitsekhovitch 2007 Modeling sawtooth stabilization by energetic ions from neutral beam injection. Phys. Plasmas, 14(7), 070703.

[14] I. T. Chapman 2011 Controlling sawtooth oscillations in tokamak plasmas. Plasma Phys. Control. Fusion 53 (1), 013001.

[15] X. Gao and the EAST team 2018 Sustained high $\beta$ Nplasmas on EAST tokamak, Physics Letters A 382(18), 1242-1246.

[16] E. D. Fredrickson, N. A. Crocker, R. E. Bell, D. S. Darrow, N. N. Gorelenkov, G. J. Kramer, S. Kubota, F. M. Levinton, D. Liu, S. S. Medley, M. Podestá, K. Tritz, R. B. White, and H. Yuh 2009 Modeling fast-ion transport during toroidal Alfvén eigenmode avalanches in National Spherical Torus Experiment. Phys. Plasmas, 16(12), 122505.

[17] E.D. Fredrickson, N.A. Crocker, D.S. Darrow, N.N. Gorelenkov, G.J. Kramer, S. 
Kubota, M. Podesta1, R.B. White, A. Bortolon, S.P. Gerhardt, R.E. Bell, A. Diallo, B. LeBlanc, F.M. Levinton and H. Yuh 2013 Fast-ion energy loss during TAE avalanches in the National Spherical Torus Experiment. Nucl. Fusion, 53(1), 013006.

[18] B. Coppi and F. Porcelli 1986 Theoretical Model of Fishbone Oscillations in Magnetically Confined Plasmas. Phys. Rev. Lett. 57(18), 2272.

[19] L. Chen, F. Zonca 2016 Physics of Alfvén waves and energetic particles in burning plasmas. Review of Modern Physics, 88(1),015008.

[20] V. Igochine 2015 Active control of Magneto-hydrodynamic Instabilities in Hot Plasmas. Springer-Verlag Berlin Heidelberg, p 116. 